\title{
An Ultrathin Endoscope with a 2.4-mm Working Channel Shortens the Esophagogastroduodenoscopy Time by Shortening the Suction Time
}

\author{
Satoshi Shinozaki ${ }^{1,2}$, Yoshimasa Miura ${ }^{2}$, Yuji Ino ${ }^{2}$, Kenjiro Shinozaki ${ }^{1}$, Alan Kawarai Lefor ${ }^{3}$ and Hironori Yamamoto ${ }^{2}$ \\ ${ }^{1}$ Department of Gastroenterology, Shinozaki Medical Clinic, Tochigi, ${ }^{2}$ Division of Gastroenterology, Department of Medicine, ${ }^{3}$ Department of \\ Surgery, Jichi Medical University School of Medicine, Shimotsuke, Japan
}

Background/Aims: Poor suction ability through a narrow working channel prolongs esophagogastroduodenoscopy (EGD). The aim of this study was to evaluate suction with a new ultrathin endoscope (EG-580NW2; Fujifilm Corp.) having a 2.4-mm working channel in clinical practice.

Methods: To evaluate in vitro suction, $200 \mathrm{~mL}$ water was suctioned and the suction time was measured. The clinical data of 117 patients who underwent EGD were retrospectively reviewed on the basis of recorded video, and the suction time was measured by using a stopwatch.

Results: In vitro, the suction time with the EG-580NW2 endoscope was significantly shorter than that with the use of an ultrathin endoscope with a 2.0 -mm working channel (EG-580NW; mean \pm standard deviation, $22.7 \pm 1.1$ seconds vs. $34.7 \pm 2.2$ seconds; $p<0.001$ ). We analyzed the total time and the suction time for routine EGD in 117 patients (50 in the EG-580NW2 group and 67 in the EG$580 \mathrm{NW}$ group). In the EG-580NW2 group, the total time for EGD was significantly shorter than that in the EG-580NW group ( $275.3 \pm 42.0$ seconds vs. $300.6 \pm 46.5$ seconds, $p=0.003$ ). In the EG-580NW2 group, the suction time was significantly shorter than that in the EG-580NW group (19.2 \pm 7.6 seconds vs. $38.0 \pm 15.9$ seconds, $p<0.001)$.

Conclusions: An ultrathin endoscope with a 2.4-mm working channel considerably shortens the routine EGD time by shortening the suction time, in comparison with an endoscope with a 2.0-mm working channel. Clin Endosc 2015;48:516-521

Key Words: Endoscopes; Suction; Early detection of cancer; Diagnosis

\section{INTRODUCTION}

Esophagogastroduodenoscopy (EGD) is routinely performed by gastroenterologists. In countries with a high prevalence of Helicobacter pylori infection, routine screening for gastric cancer by using EGD is accepted as a tolerable and short-duration procedure. Although awake transoral EGD is

Received: January 8, 2015 Revised: April 15, 2015

Accepted: April 15, 2015

Correspondence: Hironori Yamamoto

Division of Gastroenterology, Department of Medicine, Jichi Medical University School of Medicine, 3311-1 Yakushiji, Shimotsuke, Tochigi 329-0498, Japan Tel: +81-285-58-7347, Fax: +81-285-40-6598, E-mail: ireef@jichi.ac.jp

(cc This is an Open Access article distributed under the terms of the Creative Commons Attribution Non-Commercial License (http://creativecommons.org/ licenses/by-nc/3.0) which permits unrestricted non-commercial use, distribution, and reproduction in any medium, provided the original work is properly cited. the standard method for gastric cancer screening in Japan, it adversely affects the experience of patients undergoing routine EGD because of the discomfort caused by the gag reflex and pharyngeal pain. ${ }^{1}$

The development of an ultrathin endoscope with a distal diameter of $\leq 6 \mathrm{~mm}$ has enabled the insertion of the endoscope through the patients' nose. The benefit is enhanced comfort during the EGD procedure, and EGD is well tolerated regardless of the insertion route. EGD with an ultrathin endoscope without sedation is a safer and more comfortable procedure than awake conventional EGD., The use of a safe and tolerable EGD technique will contribute to the early detection of gastric cancer.

One of the major limitations of using an existing ultrathin endoscope is the narrow working channel $(2.0 \mathrm{~mm})$ that leads to poor suction ability and the inability to perform therapeu- 

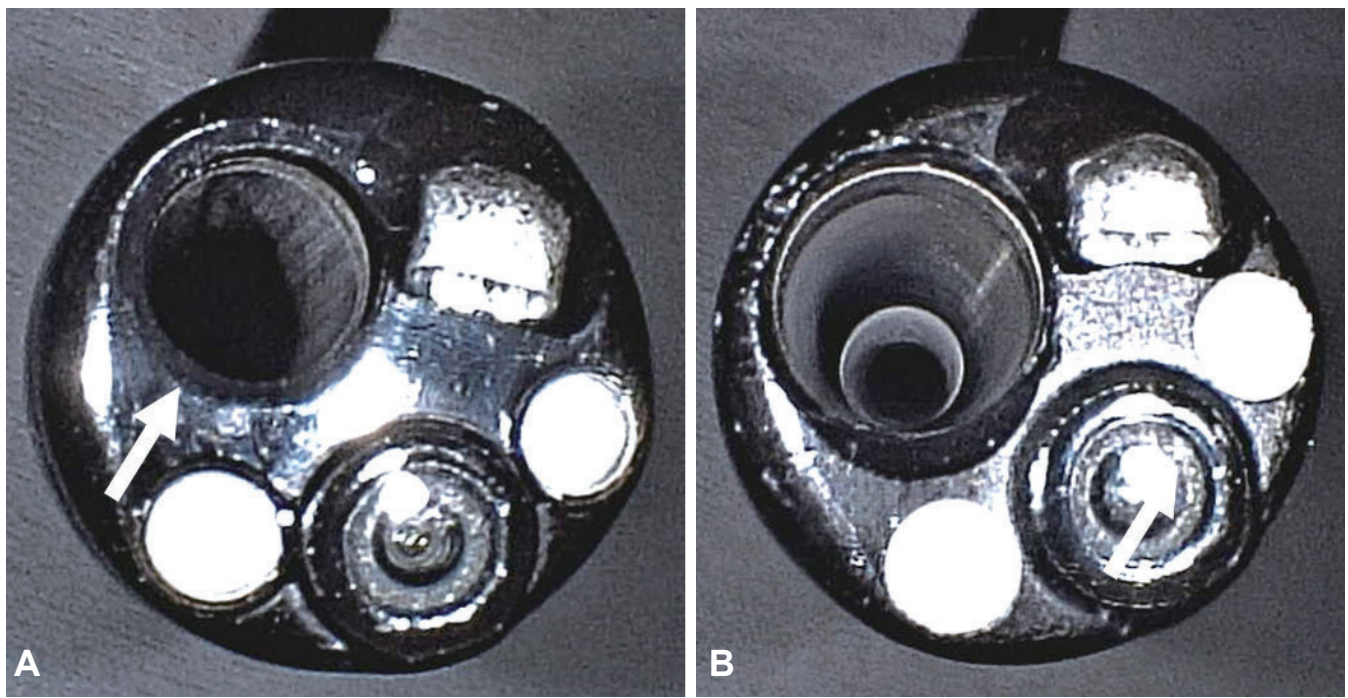

Fig. 1. The tip of the ultrathin endoscopes: (A) EG-580NW (Fujifilm Corp.) with a 2.0-mm working channel (arrow) and 5.9-mm distal end diameter, and (B) EG580NW2 with a 2.4-mm working channel (arrow) and 5.8-mm distal end diameter. The common specifications for the two endoscopes are as follows: field of view, $140^{\circ}$; flexible portion diameter, $5.9 \mathrm{~mm}$; and total length, $1,400 \mathrm{~mm}$.

tic interventions. As a result, the EGD time is significantly longer than that with the conventional transoral EGD. ${ }^{1}$ Furthermore, the longer suction time during EGD may cause discomfort for both the patients and the endoscopists.

A new ultrathin endoscope with a 2.4-mm working channel (EG-580NW2; Fujifilm Corp., Tokyo, Japan) was made available in Japan in October 2013. The diameter of the working channel is 1.2 times larger than that of the existing ultrathin endoscope (EG-580NW, with a 2.0-mm working channel; Fujifilm) (Fig. 1). To date, there are no studies focusing on the suction ability through the working channel of the ultrathin endoscope. Few studies have reported on the suction ability of ultrathin endoscopes. Although these studies provide the endoscopist's evaluation, they neither report the suction time nor compare the working channel. ${ }^{4,5}$ Furthermore, it is not known how an ultrathin endoscope with a wide working channel influences EGD when used in the human stomach, which has a complex structure and contains viscous fluid. We have used this new endoscope at our institute since April 2014 for routine EGD procedures. The aim of this study was to assess the suction ability of the EG-580NW2 endoscope in an experimental setting, and to evaluate the effect of using the EG-580NW2 on the conduct of routine EGD in patients, which may be affected by the complex structure of the human stomach and the presence of viscous gastric fluid.

\section{MATERIALS AND METHODS}

\section{Specifications of the ultrathin endoscopes}

We used a new ultrathin endoscope with a 2.4-mm working channel (EG-580NW2), and the existing ultrathin endoscope with a $2.0-\mathrm{mm}$ working channel (EG-580NW) as the control (Fig. 1). These two endoscopes are almost the same in all aspects, except for the diameter of the working channel (Fig. 1).

\section{In vitro suction procedure}

To evaluate the in vitro suction ability of both endoscopes, we prepared a beaker with $200 \mathrm{~mL}$ water, placed the tip of the endoscope at the bottom, and measured the time needed to completely aspirate the water.

\section{Patients and the EGD procedure}

We conducted a retrospective study of patients who underwent EGD at the Shinozaki Medical Clinic. The exclusion criteria included patients who had undergone surgical resection of the stomach, those who required a biopsy, those who required extensive flushing because of the presence of bubbles, or those with residual food in the stomach. The clinical data were retrospectively collected from medical records, photographs, and video recordings. $H$. pylori infection was evaluated by testing for anti-H. pylori immunoglobulin G antibody in the serum. All EGD procedures were recorded on video. The Institutional Review Board of Shinozaki Medical Clinic approved this retrospective study on August 23, 2014.

We began using the EG-580NW2 endoscope in April 2014 in addition to using an existing EG-580NW endoscope. These two instruments were used alternately because the clinic has only these two endoscopes for use in EGD. From April 19 to July 24, 2014, 200 consecutive EGD procedures were performed in 200 patients at Shinozaki Medical Clinic. We used the EG-580NW endoscope in 114 patients and the EG- 
$580 \mathrm{NW} 2$ endoscope in 86 patients. The EG-580NW2 was not used for 2 weeks during the study period because of required maintenance. On the basis of the above criteria, 83 patients were excluded, including those with gastric resection $(n=7)$, biopsy $(n=53)$, flushing to remove extensive bubbles $(n=42)$, and the presence of residual food $(n=2)$ (several patients met more than one criteria). Finally, the data for 117 patients were included in the final analysis.

The fasting time before routine EGD was $>12$ hours, and oral intake was prohibited on the day of EGD. Just before undergoing EGD, the patients ingested $16 \mathrm{~mL}$ water with $80 \mathrm{mg}$ dimethylpolysiloxane (Gascon; Kissei, Tokyo, Japan). All EGD procedures were performed between 9:00 AM and 12:00 PM. No sedation was used. The first-choice insertion route was per nasal, and the per-oral route was chosen when per-nasal insertion was difficult because of a narrow nasal cavity or patient discomfort. The time for conversion to the per-oral route was not included in the total procedure time. During these routine EGD procedures, observation was done routinely, including that of the esophagus, duodenum, and stomach. After the observation of the duodenum, the antrum was observed and gastric fluid was suctioned. Bubbles were washed out by flushing water from the working channel, if necessary. Suctioning of gastric fluid was done with a retroflexed endoscope so that the endoscope was parallel to the gastric wall and did not suction the gastric mucosa. After completely aspirating the fluid, the entire stomach was carefully evaluated. The total time and the suction time were measured by using a stopwatch while reviewing the video. The observation time was calculated by subtracting the suction time from the total time. All EGD procedures in this study were performed by the first author (SS). No adverse events occurred after the EGD procedures.

\section{Statistical analysis}

Categorical data were compared by using Fisher exact test. Data with a normal distribution were compared with a paired Student $t$-test. Differences were considered to be statistically significant when $p<0.05$.

\section{RESULTS}

\section{Improved in vitro suctioning with the EG-580NW2 endoscope}

We assessed the time for in vitro suctioning with the EG580NW and EG-580NW2 endoscopes. For $200 \mathrm{~mL}$ water, the suction time for the EG-580NW2 was significantly shorter than that of the EG-580NW ( $p<0.001)$ (Fig. 2). The suction time of the EG-580NW2 was approximately 1.5 times faster than that of the EG-580NW in vitro.

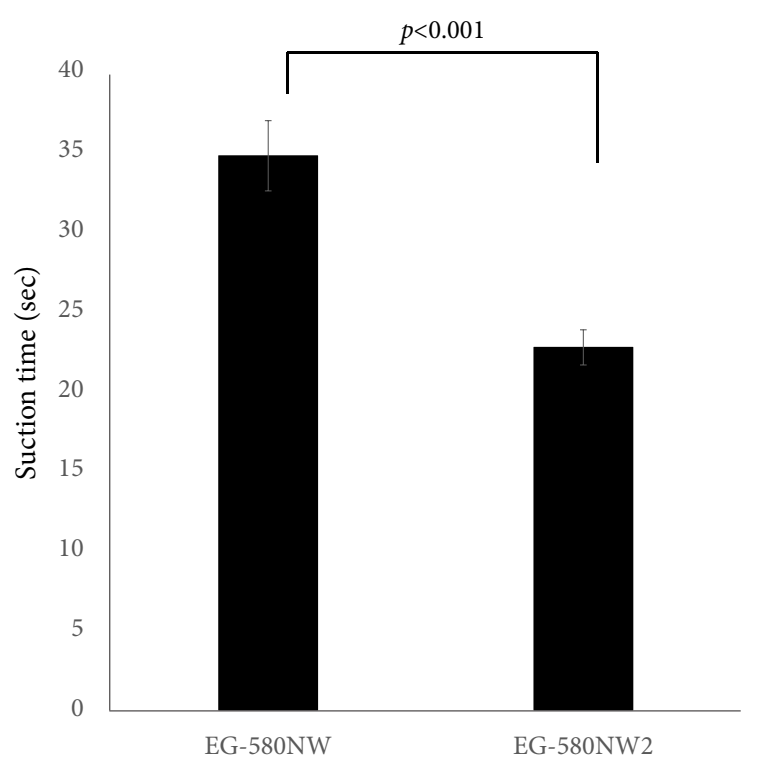

Fig. 2. Comparison of the time needed to aspirate $200 \mathrm{~mL}$ water in vitro by using the EG-580NW and EG-580NW2 (Fujifilm Corp.) endoscopes. The values are expressed as mean $\pm S D$.

\section{Patient characteristics and EGD procedures}

We analyzed the total time and the suction time while performing routine EGD in 117 patients, including 67 patients who underwent EGD with the EG-580NW (57\%) and 50 patients with the EG-580NW2 (43\%). The baseline characteristics of the two groups were compared (Table 1), including gender, age, use of acid-suppressing medications, intubation route, $H$. pylori infection, and endoscopic findings. There were no significant differences except for the proportion of male patients. Despite careful review of the data, no explanation could be given for the preponderance of male patients.

\section{Shortened total EGD time and suction time with the EG-580NW2 endoscope}

We measured the total time for performing EGD and the time required to aspirate gastric fluid (Table 2), and calculated the proportion of suction time relative to the total time for performing EGD. In the EG-580NW2 group, the total time for EGD was significantly shorter, by approximately $25 \mathrm{sec}$ onds, than that of the EG-580NW group ( $p=0.003$ ) (Table 2). The suction time in the EG-580NW2 group was almost half of that of EG-580NW group, and the difference was statistically significant $(p<0.001)$. The proportion of the suction time was significantly lower in the EG-580NW2 group than in the EG$580 \mathrm{NW}$ group $(p<0.001)$; the mean proportion was $<10 \%$ in the EG-580NW2 group. The observation time was almost the same between the two groups ( $p=0.419)$. Therefore, the EG$580 \mathrm{NW} 2$ endoscope significantly shortened the total time for EGD by shortening the suction time without decreasing the total observation time. 
Table 1. Clinical Data of Patients and Endoscopic Findings

\begin{tabular}{|c|c|c|c|}
\hline Variable & EG-580NW $(n=67)$ & EG-580NW2 $(n=50)$ & $p$-value \\
\hline Male sex & $40(60)$ & $18(36)$ & 0.015 \\
\hline Age, yr & $62 \pm 13$ & $59 \pm 13$ & 0.264 \\
\hline \multicolumn{4}{|l|}{ Indications } \\
\hline Screening & $25(37)$ & $18(36)$ & 0.280 \\
\hline Follow-up for atrophic gastritis & $16(24)$ & $10(20)$ & 0.394 \\
\hline Follow-up for specific lesion ${ }^{\text {a) }}$ & $12(18)$ & $8(16)$ & 0.494 \\
\hline Investigation for symptom ${ }^{\text {b) }}$ & $7(10)$ & $11(22)$ & 0.074 \\
\hline Abnormality of the GI series & $3(4)$ & $1(2)$ & 0.427 \\
\hline Patient's desire & $2(3)$ & $2(4)$ & 0.574 \\
\hline Positive Helicobacter pylori IgG & $1(2)$ & 0 & 0.573 \\
\hline Investigation for anemia & $1(2)$ & 0 & 0.573 \\
\hline \multicolumn{4}{|l|}{ Acid suppression drug intake } \\
\hline None & $22(33)$ & $11(22)$ & 0.219 \\
\hline Histamine 2 receptor antagonist & $17(25)$ & $16(32)$ & 0.533 \\
\hline Proton pomp inhibitor & $28(42)$ & $23(46)$ & 0.708 \\
\hline Intubation route (per nasal) & $63(94)$ & $45(90)$ & 0.494 \\
\hline \multicolumn{4}{|l|}{ H. pylori infection } \\
\hline Infected & $12(18)$ & $6(12)$ & 0.445 \\
\hline Not infected without eradication & $35(52)$ & $29(58)$ & 0.577 \\
\hline Not infected after eradication & $15(22)$ & $14(28)$ & 0.521 \\
\hline Not assessed & $5(8)$ & $1(2)$ & 0.236 \\
\hline \multicolumn{4}{|l|}{ Endoscopic findings } \\
\hline Gastric atrophy & $45(67)$ & $33(66)$ & 1.000 \\
\hline Intestinal metaplasia & $18(27)$ & $7(14)$ & 0.113 \\
\hline Fundic gland polyp & $11(16)$ & $11(22)$ & 0.480 \\
\hline Hyperplastic polyp & $1(2)$ & $1(2)$ & 1.000 \\
\hline Gastric ulcer scar & $1(2)$ & 0 & 1.000 \\
\hline Duodenal ulcer scar & $5(8)$ & $3(6)$ & 1.000 \\
\hline
\end{tabular}

Values are presented as number (\%) or mean \pm SD.

GI, gastrointestinal; IgG, immunoglobulin G.

${ }^{a)}$ Regurgitational esophagitis, polyps, ulcer scar, postoperative scar, varix, or submucosal tumor; ${ }^{\text {b) }}$ Abdominal symptoms, heartburn, or melena.

Table 2. Comparison of Suction Times in Routine Esophagogastroduodenoscopy

\begin{tabular}{lccc}
\hline Variable & EG-580NW & EG-580NW2 & p-value \\
\hline Total time, sec & $300.6 \pm 46.5$ & $275.3 \pm 42.0$ & 0.003 \\
Suction time, sec & $38.0 \pm 15.9$ & $19.2 \pm 7.6$ & $<0.001$ \\
Proportion of suction time (mean), $\%^{\mathrm{a})}$ & 12.6 & 7.1 & - \\
Observation time, sec & $262.5 \pm 41.9$ & $256.2 \pm 42.4$ & 0.419 \\
\hline
\end{tabular}

Values are presented as mean \pm SD.

${ }^{\text {a) }}$ The proportion of suction time is calculated as suction time/total time. 


\section{DISCUSSION}

The development of an ultrathin endoscope has allowed patients to undergo comfortable and tolerable routine EGD without sedation. ${ }^{6}$ However, the long examination time caused by the narrow working channel became a problem despite the advantages of this instrument. ${ }^{3,7}$ The present study showed the superiority of the ultrathin endoscope with a 2.4-mm working channel over an endoscope with a 2.0-mm channel both in vitro and in clinical practice. In the routine EGD procedure, endoscopy with a 2.4-mm working channel shortened the total EGD time by shortening the suction time by approximately 25 seconds. The shortened suction time did not influence the total observation time.

There are few reports about the suction time when using an ultrathin endoscope. Dumortier et al. ${ }^{5}$ compared ultrathin endoscopes with different diameters $(4.9 \mathrm{~mm}$ vs. $5.9 \mathrm{~mm})$. They reported improved feasibility and patient tolerance with the 4.9-mm endoscope. Although they also reported the endoscopist's subjective rating on suction ability, the actual suction time was not reported and the diameter of the working channel was $2.0 \mathrm{~mm}$ in both endoscopes. To our knowledge, the present study is the first to compare the actual suction times between an ultrathin endoscope with a 2.4$\mathrm{mm}$ and that with a $2.0-\mathrm{mm}$ working channel. Trevisani et al. ${ }^{4}$ reported a randomized study with both conventional and ultrathin endoscopes with a $2.0-\mathrm{mm}$ working channel. The endoscopist's evaluation of the suction with the ultrathin endoscope was significantly worse than that with the conventional endoscope. The procedure time to perform transnasal EGD with the ultrathin endoscope with a $2.0-\mathrm{mm}$ working channel was significantly longer, by about 0.6 minute, than that for the conventional EGD with an endoscope with a 2.8-mm working channel. In the present study, the wider working channel in the ultrathin endoscope shortened the total time by about 0.4 minute. The $2.4-\mathrm{mm}$ working channel may bring the time to perform EGD closer to that when using a conventional endoscope. A prospective study on the use of conventional and ultrathin endoscopes with 2.4-mm working channels is necessary to resolve this issue. The EG$580 \mathrm{NW} 2$ resulted in a $35 \%$ reduction in suction time in vitro and a $49 \%$ reduction in suction time in clinical use compared with the EG-580NW. As the flow rates were proportional to the area, and the ratio of the area of the channels of the two endoscopes was 1.44:1, this difference is at the expected order of magnitude. The difference in suction rates between the in vitro and clinical settings is partially explained by the higher viscosity of gastric fluid compared with water. Unlike the EG-580NW2, the EG-580NW required prolonged suction time during EGD procedures when high-viscosity fluid oc- cluded the narrow working channel. The long suction time needed to aspirate a high-viscosity fluid may prevent the adequate and precise observation during EGD. Additionally, to obtain certain data for suctioning, we excluded patients who underwent extensive gastric flushing to eliminate bubbles. However, the EG-580NW2 may demonstrate an improved ability in such a situation if there is large amount of fluid in the stomach.

Although many EGD procedures with an ultrathin endoscope are performed worldwide, the suction ability has not been previously evaluated in a quantitative manner, despite it being an important issue. Poor suction ability during EGD not only influences the examination time but also causes frustration to the endoscopist. This study shows that a $0.4-$ $\mathrm{mm}$ difference in the working channel diameter shortens the suction time and the total time for EGD. The EG-580NW2 endoscope may contribute to decreasing the difficulty for the endoscopist as well as to improving the quality of EGD. We also recognize the limitations of this study, including the following: (1) its retrospective design; (2) the female predominance in the EG-580NW2 group might have resulted in a bias; (3) the study reflects the experience of a single endoscopist; (4) there is no comparison group patients who underwent conventional endoscopy; and (5) patient comfort was not assessed.

In conclusion, a new ultrathin endoscope with a $2.4-\mathrm{mm}$ working channel can shorten the time needed to perform routine EGD significantly by shortening the suction time, compared with endoscopes that have a $2.0-\mathrm{mm}$ working channel. We believe that the shortened suction time will improve the endoscopist's experience and facilitate adequate observation during routine EGD.

\section{Conflicts of Interest}

Author HY has a consultant relationship with Fujifilm Corp., and has received honoraria, grants, and royalties from the company. He has patents for double-balloon endoscope and endoscopic submucosal dissection devices produced by the Fujifilm Corp. The funding source had no role in the design, practice, or analysis of this study. The other authors have no financial conflicts of interest. No funding was provided for this study.

\section{REFERENCES}

1. Kadayifci A, Atar M, Parlar S, Balkan A, Koruk I, Koruk M. Transnasal endoscopy is preferred by transoral endoscopy experienced patients. J Gastrointestin Liver Dis 2014;23:27-31.

2. Murata A, Akahoshi K, Motomura Y, et al. Prospective comparative study on the acceptability of unsedated transnasal endoscopy in younger versus older patients. J Clin Gastroenterol 2008;42:965-968.

3. Atar M, Kadayifci A. Transnasal endoscopy: technical considerations, advantages and limitations. World J Gastrointest Endosc 2014;6:41-48.

4. Trevisani L, Cifalà V, Sartori S, Gilli G, Matarese G, Abbasciano V. Unsedated ultrathin upper endoscopy is better than conventional endoscopy in routine outpatient gastroenterology practice: a randomized trial. 
World J Gastroenterol 2007;13:906-911.

5. Dumortier J, Josso C, Roman S, et al. Prospective evaluation of a new ultrathin one-plane bending videoendoscope for transnasal EGD: a comparative study on performance and tolerance. Gastrointest Endosc 2007;66:13-19
6. Garcia RT, Cello JP, Nguyen MH, et al. Unsedated ultrathin EGD is well accepted when compared with conventional sedated EGD: a multicenter randomized trial. Gastroenterology 2003;125:1606-1612.

7. ASGE Technology Committee, Rodriguez SA, Banerjee S, et al. Ultrathin endoscopes. Gastrointest Endosc 2010;71:893-898. 